\title{
Phase separation in imbalanced fermion superfluids beyond the mean-field approximation
}

\author{
J. Tempere, ${ }^{*}$ S. N. Klimin, ${ }^{\dagger}$ and J. T. Devreese ${ }^{\ddagger}$ \\ Theoretische Fysica van de Vaste Stoffen (TFVS), Universiteit Antwerpen, B-2020 Antwerpen, Belgium \\ (Received 26 December 2007; revised manuscript received 16 June 2008; published 21 August 2008)

\begin{abstract}
The density distributions of the two components of a trapped, ultracold Fermi gas with population imbalance reveal the effect of imbalance on superfluid pairing. We develop a path-integral derivation of the density, that takes into account both fluctuations beyond mean-field and effects of nonzero temperature. The calculated density profiles compare favorably to the measured density profiles, and illustrate the necessity to incorporate both quantum fluctuations and finite temperature effects. The temperature dependence of the density profiles, especially near the superfluid-normal interface, allows for determining the temperature of the superfluid core in current experiments.
\end{abstract}

DOI: 10.1103/PhysRevA.78.023626

PACS number(s): 03.75.Lm, 05.30.Fk, 03.75.Ss

Recent progress in the trapping of cold atoms has attracted great interest related to a wide variety of fields: Condensed matter physics, atomic, molecular, and optical physics, astrophysics and physics of quark and nuclear matter. There exists a deep analogy between the dynamics of cold atoms, astrophysical systems, nuclear and plasma systems [1-4]. The possibility to tune the effective interaction strength and to control populations of different spin states provides a unique opportunity to investigate various phenomena of interacting many-body systems. Imbalanced Fermi superfluids are in particular relevant for color superconductivity in dense quark matter $[5,6]$ and for neutronproton pairing in asymmetric nuclear matter [7].

In experiments [8-13], a phase separation between the superfluid and normal component of an interacting Fermi gas of cold atoms with unequal spin populations has been observed. These experiments identify a shell structure of the fermion cloud, in which a superfluid core is surrounded with a Fermi gas in the normal state.

The mean-field approach provides a convincing qualitative explanation of the phase separation of imbalanced cold fermion atoms in a trap [14-16]. Mean field predicts a superfluid core (which at $T=0$ is completely unpolarized) surrounded with an imbalanced normal phase, and a discontinuous behavior of the majority and minority component densities at the phase boundary. The theoretical study of a trapped strongly interacting Fermi gas in the unitarity limit at the zero temperature [17] shows a good agreement of the radii for the majority and minority components with the experimental results of Ref. [11]. However, by contrast with experiments, the boundary between the two phases provided by the mean-field approach (see Refs. [14-17] and the meanfield results below in the present work) is very sharp both for elongated and oblate traps, i.e., independently of a trap an-

\footnotetext{
*Also at the Lyman Laboratory of Physics, Harvard University, Cambridge, MA 02138.

†Permanent address: Department of Theoretical Physics, State University of Moldova, str. A. Mateevici 60, MD-2009 Kishinev, Republic of Moldova.

${ }^{\ddagger}$ Also at Technische Universiteit Eindhoven, P.B. 513, $5600 \mathrm{MB}$ Eindhoven, The Netherlands.
}

isotropy, while experimentally observed phase boundaries are rather smoothed in the case of Ref. [10]. Here, we describe quantitatively this smoothing by taking into account effects beyond the mean-field approximation, at nonzero temperature. The density profiles derived in this work offer the possibility to determine the temperature of the superfluid by a fitting procedure, all other parameters being fixed by the experimental configuration.

Recent experiments investigating pairing of cold atoms are realized in the crossover regime between weak coupling, where the Bardeen-Cooper-Schrieffer (BCS) theory holds, and strong coupling where the system is well described as a Bose-Einstein condensate (BEC) of molecules. In the BCSBEC crossover regime, the critical temperature $T_{c}$ of the superfluid phase transition is substantially lowered with respect to $T_{c}$ obtained within the mean-field approach. The lowering of $T_{c}$ is due to Gaussian fluctuations about the saddle point. The fluctuations also influence other parameters of the fermion system, such as the density. In addition to fluctuations, other factors also influence the density profiles calculated for cold Fermi gases: The possible violation of the local density approximation (LDA) in highly anisotropic traps observed by Hulet and co-workers [11-13] or the occurrence of nontrivial phases such as Fulde-Ferrell-Larkin-Ovchinnikov state as reported by Yoshida and Yip [18]. These mechanisms can be described using the method outlined below, but this is beyond the scope of the present treatment, which focuses on the role of fluctuations. High-resolution density distributions of cold ${ }^{6} \mathrm{Li}$ atoms measured by Ketterle and co-workers [10] allow us to perform a quantitative comparison between the experiment and theory.

There are different techniques to treat degenerate Fermi gases in the BCS-BEC crossover regime. The diagrammatic technique is applied by Perali et al. [19], Chen et al. [20], and Taylor et al. [21] to extend the results obtained by Nozières and Schmitt-Rink (NSR) [22] for $T=T_{c}$ to arbitrary temperatures. The NSR-like scheme [23] provides the equation of state which is in excellent agreement with Monte Carlo calculations within the whole range of the BCS-BEC crossover at finite temperatures. This approach is also capable to show the universal thermodynamics of strong interacting fermions [24,25].

In order to investigate the phase separation of an interact- 
ing Fermi gas in a trap, we use the path-integral formalism [26-28]. The path-integral method is attractive because it allows us to obtain reliable parameters for interacting fermions in the whole range of the coupling strength. However, it is necessary to go beyond the low-temperature approximation of Refs. [27,28], because the contribution to the density due to fluctuations is sensitive to the temperature variations, even in the low-temperature region.

In the present work, the path-integral approach is extended to the case of arbitrary finite temperature and imbalanced spin populations, and applied to calculate fluctuation contributions to the density. We consider a two-component Fermi gas with the spin states $|+\rangle$ and $|-\rangle$ in an anisotropic parabolic trap within the LDA. The coordinate dependence of the density of fermions in a given spin state is determined through the chemical potential

$$
\mu_{ \pm}(\mathbf{r})=\mu_{ \pm}(0)-\frac{m}{2}\left[\omega_{1}^{2}\left(x^{2}+y^{2}\right)+\omega_{2}^{2} z^{2}\right]
$$

characterized by the confinement frequencies $\omega_{1}$ and $\omega_{2}$. For given values of the local density $n=n_{+}+n_{-}$and of the local density difference $\delta n=n_{+}-n_{-}$, the averaged chemical potential $\mu \equiv\left(\mu_{+}+\mu_{-}\right) / 2$, the imbalance potential $\zeta \equiv\left(\mu_{+}-\mu_{-}\right) / 2$ and the gap parameter $\Delta$ are found as a joint solution of the gap equation and of the two number equations. The thermodynamic potential and therefore the densities are determined in the quadratic approximation with respect to the quantum fluctuations about the saddle point for arbitrary temperatures and taking into account the population imbalance.

Within the NSR scheme, the Gaussian fluctuations do not feedback into the saddle-point equation for the functional integral, and the fluctuations only contribute to the fermion density and to the number equation. Hence, the gap equation takes the same form as in the mean-field approximation. As shown in Ref. [28], this is a natural approximation within the path-integral formalism.

For a balanced gas, the saddle-point thermodynamic potential has a single minimum, which results in the gap equation. For an imbalanced gas, however, the saddle-point thermodynamic potential can have two minima at $\Delta=0$ and at $\Delta \neq 0$ [29]. Therefore, the phase boundary for an imbalanced Fermi gas in a trap cannot be determined from the gap equation, as well as from the Thouless criterion [30], because they both allow one to find only a local minimum of the variational problem for the thermodynamic potential [30]. As a natural extension of the NSR scheme to an imbalanced Fermi gas, we find coordinate-dependent values of the gap parameter through the straightforward minimization of the saddle-point thermodynamic potential [31],

$$
\begin{aligned}
\frac{\Omega_{s p}}{V}= & -\int \frac{d \mathbf{k}}{(2 \pi)^{3}}\left(\frac{1}{\beta} \ln \left(2 \cosh \beta \zeta+2 \cosh \beta E_{\mathbf{k}}\right)\right. \\
& \left.-\varepsilon_{\mathbf{k}}-\frac{|\Delta|^{2}}{2 k^{2}}\right)-\frac{|\Delta|^{2}}{8 \pi a_{s}},
\end{aligned}
$$

where $a_{s}$ is the scattering length, $\varepsilon_{\mathbf{k}}=k^{2}-\mu$ is the fermion energy, and $E_{\mathbf{k}}=\sqrt{\varepsilon_{\mathbf{k}}^{2}+|\Delta|^{2}}$ is the Bogolubov excitation en- ergy. We use the units in which $\hbar=1, m=1 / 2$, and the Fermi energy $E_{F}=1$.

The fermion densities are a sum of mean-field and fluctuation contributions. The current finite temperature implementation of the extended NSR scheme is similar to that used in studying $d$-wave pairing in Ref. [31]. For the case of the $s$-wave pairing mechanism, we find that the fluctuation contribution $n_{f l}$ to the total density $n$ is given by

$$
\begin{aligned}
n_{f l}= & -\int \frac{d \mathbf{q}}{(2 \pi)^{3}}\left(\frac{1}{\pi} \int_{-\infty}^{\infty} \operatorname{Im} \frac{J(\mathbf{q}, \omega+i \gamma)}{e^{\beta(\omega+i \gamma)}-1} d \omega\right. \\
& \left.+\frac{1}{\beta} \sum_{n=-n_{0}}^{n_{0}} J\left(\mathbf{q}, i \Omega_{n}\right)\right),
\end{aligned}
$$

with $\beta$ the inverse to the temperature. Here, $n_{0}$ is an arbitrary positive integer, and the parameter $\gamma$ lies between two bosonic Matsubara frequencies $\Omega_{n_{0}}<\gamma<\Omega_{n_{0}+1}, \quad \Omega_{n}$ $\equiv 2 \pi n / \beta$. For the computation, a fast convergence of the integral over $\omega$ in (3) is achieved when $\gamma=(2 n+1) \pi / \beta$, and an integer $n_{0}$ is chosen in such a way that $\gamma \sim 1$. The function $J(\mathbf{q}, z)$ of complex argument $z$ is

$$
J(\mathbf{q}, z)=\frac{M_{1,1}(\mathbf{q},-z) \frac{\partial M_{1,1}(\mathbf{q}, z)}{\partial \mu}-M_{1,2}(\mathbf{q},-z) \frac{\partial M_{1,2}(\mathbf{q}, z)}{\partial \mu}}{M_{1,1}(\mathbf{q}, z) M_{1,1}(\mathbf{q},-z)-M_{1,2}^{2}(\mathbf{q}, z)} .
$$

The fluctuation contribution $\delta n_{f l}$ to the density difference $\delta n$ is given by the same expression as (3) with replacing the derivatives $\partial / \partial \mu$ in the function $J(\mathbf{q}, z)$ by $\partial / \partial \zeta$. The matrix elements $M_{j, k}(\mathbf{q}, z)$ are given by

$$
\begin{aligned}
M_{1,1}(\mathbf{q}, z)= & \int \frac{d \mathbf{k}}{(2 \pi)^{3}}\left[\frac{1}{2 k^{2}}+\frac{\sinh \beta E_{\mathbf{k}}}{2 E_{\mathbf{k}}\left(\cosh \beta E_{\mathbf{k}}+\cosh \beta \zeta\right)}\right. \\
& \times\left(\frac{\left(z-E_{\mathbf{k}}+\varepsilon_{\mathbf{k}+\mathbf{q}}\right)\left(E_{\mathbf{k}}+\varepsilon_{\mathbf{k}}\right)}{\left(z-E_{\mathbf{k}}+E_{\mathbf{k}+\mathbf{q}}\right)\left(z-E_{\mathbf{k}}-E_{\mathbf{k}+\mathbf{q}}\right)}\right. \\
& \left.\left.-\frac{\left(z+E_{\mathbf{k}}+\varepsilon_{\mathbf{k}+\mathbf{q}}\right)\left(E_{\mathbf{k}}-\varepsilon_{\mathbf{k}}\right)}{\left(z+E_{\mathbf{k}}-E_{\mathbf{k}+\mathbf{q}}\right)\left(z+E_{\mathbf{k}+\mathbf{q}}+E_{\mathbf{k}}\right)}\right)\right]-\frac{1}{8 \pi a_{s}},
\end{aligned}
$$

$$
\begin{aligned}
M_{1,2}(\mathbf{q}, z)= & -|\Delta|^{2} \int \frac{d \mathbf{k}}{(2 \pi)^{3}} \frac{\sinh \beta E_{\mathbf{k}}}{2 E_{\mathbf{k}}\left(\cosh \beta E_{\mathbf{k}}+\cosh \beta \zeta\right)} \\
& \times\left(\frac{1}{\left(z-E_{\mathbf{k}}+E_{\mathbf{k}+\mathbf{q}}\right)\left(z-E_{\mathbf{k}}-E_{\mathbf{k}+\mathbf{q}}\right)}\right. \\
& \left.+\frac{1}{\left(z+E_{\mathbf{k}}-E_{\mathbf{k}+\mathbf{q}}\right)\left(z+E_{\mathbf{k}}+E_{\mathbf{k}+\mathbf{q}}\right)}\right)
\end{aligned}
$$

These expressions incorporate not only the particle-pair and hole-pair excitations, but also particle-hole excitations, so that in the limit $\Delta \rightarrow 0$ one obtains an interacting Fermi gas rather than the ideal gas.

The phase transitions of the imbalanced fermion gas were analyzed using phase diagrams for various thermodynamic variables (see Refs. [32-36]). The finite-temperature phase diagram for pressure and/or temperature $[37,38]$ is probably 
the first phase diagram which includes finite temperature and fluctuation effects as well as trap inhomogeneity. At a fixed scattering length $a_{s}$, the temperature $T_{0}$ of the tricritical point separates two types of the phase transition as follows. For $T \geqslant T_{0}$, the relative population imbalance $\delta n / n$ is continuous across the phase transition, so that the latter one is of the second order. On the contrary, at $T<T_{0}$, the relative population imbalance changes discontinuously at the phase transition, so that the latter one is of the first order [29]. As a result, there exists the region where a uniform normal or superfluid state cannot exist, and therefore in this region phase separation occurs $[32,35,36]$.

The effect of fluctuations beyond mean-field on the phase diagram is not completely resolved $[37,38]$, although the effect of Gaussian fluctuations on the locus of the tricritical points was studied in [32]. In this contribution, rather than focusing on the hard task of correcting the mean-field phase diagram, we will investigate the effects of fluctuations on the density profiles, where the fluctuation contribution is straightforwardly obtained from Eq. (3).

The phase separation exists at temperatures lower than the temperature of the tricritical point. Typical temperatures of the experiments on the phase separation of an imbalanced mixture of cold atoms are estimated to be as low as $T$ $\sim 0.1 T_{F}$, where $T_{F}$ is the Fermi temperature $T_{F}=E_{F} / k_{B}$. Therefore, the phase transition at the boundary of the superfluid core in the experiments $[10,11]$ is expected to be of the first order. In order to get a definite answer to this question, we investigate the distributions of the gap parameter and of the density for a fermion gas in an anisotropic parabolic trap taking into account Gaussian fluctuations and using the parameters typical for the conditions in the experiment by Ketterle and co-workers [10].

In Fig. 1(a), we plot one-dimensional (1D) profiles $\delta \tilde{n}$ obtained by the integration of $\delta n$ over $y$ and $z$ coordinates [corresponding to the profile of the integrated density difference from Fig. 3(d) of Ref. [10]]. Figure 1(b) shows the three-dimensional (3D) distribution profiles along the lateral direction for the density difference, compared with the experimental distribution profiles from Ref. [10]. Both with and without fluctuations, there is a spatial phase separation, where a superfluid core is surrounded by a normal phase. In the trap at a sufficiently low temperature the fermion system is almost exactly balanced in the core and almost completely polarized outside the core. Local densities for both majority and minority components change their values sharply when passing the phase boundary. Correspondingly, the total fermion density and the local density difference reveal discontinuities at the phase boundary. Those discontinuities are a consequence of the fact that the phase transition is of the first order.

Within the BCS-BEC crossover regime, the Gaussian fluctuations lead to a substantial decrease of the core radius with respect to that obtained in the mean-field approximation. The fluctuations reduce the amplitude of the discontinuous change of the density difference and provide a partially smoothed (with a relatively small discontinuity) density profile near the phase boundary. A smoothing of the radial density profiles owing to fluctuations was also obtained by Chien et al. [37,38]. The profile for $\delta n$, obtained taking into

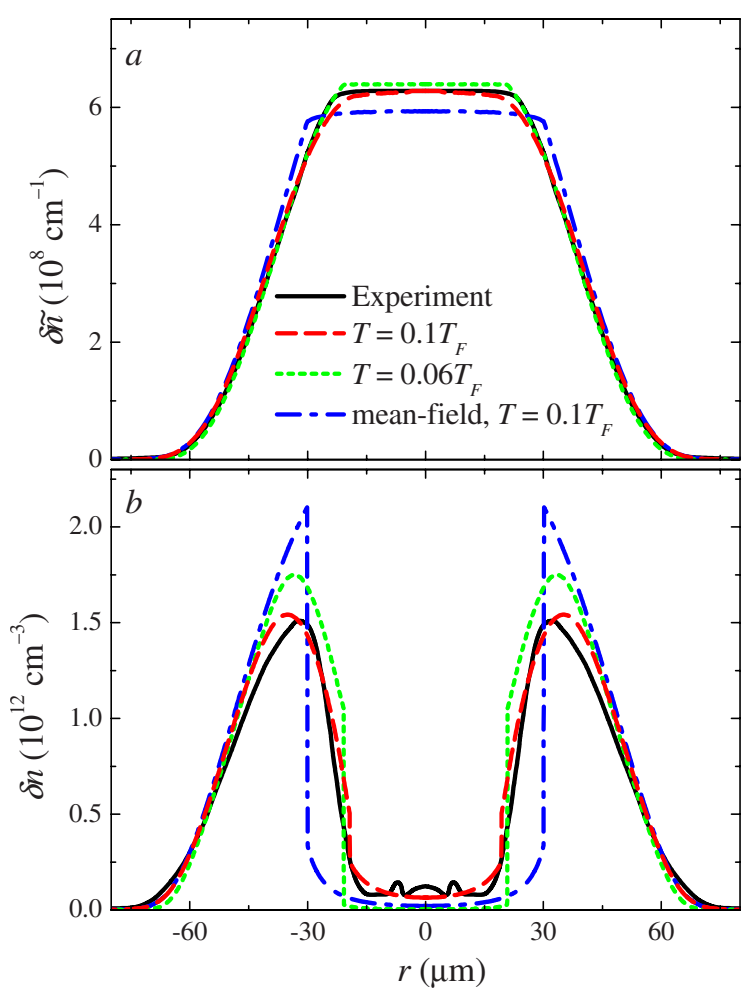

FIG. 1. (Color online) (a) The distribution profiles for the integrated density difference compared with the experimental data (full curve) from Fig. 3(d) of Ref. [10]. (b) The 3D distribution profiles along the lateral direction for the local density difference compared with the experimental 3D distribution profile (full curve) from Fig. 3(e) of Ref. [10]. Dashed and dotted curves correspond to the present approach taking into account Gaussian fluctuations, for $T$ $=0.1 T_{F}$ and $T=0.06 T_{F}$, respectively. Dotted-dashed curves correspond to the mean-field approach.

account fluctuations, lies drastically closer to the experimental radial profile [10] for the local population imbalance than the mean-field result. As for the radial distributions, the 1D profiles of the integrated density difference calculated taking into account Gaussian fluctuations are in better agreement with the experiment [10] than the mean-field results in what regards the core size and the density profile. With respect to the present approach and to the experiment, the mean-field approximation overestimates the size of the superfluid core and the magnitude of the jump of the density difference at the phase boundary.

The 3D distribution profiles along the lateral direction for the gap parameter calculated within the mean-field approximation and taking into account Gaussian fluctuations are plotted in Fig. 2. As seen from the comparison of the spatial distributions of the gap parameter obtained within the meanfield approach and within the NSR scheme, the fluctuations reduce both the core radius and the values of the gap parameter inside the core. At the phase boundary between the superfluid and normal phases, the gap parameter discontinuously changes its magnitude. When taking fluctuations into account, the first-order phase transition between the superfluid and normal states occurs at a higher magnitude of the gap parameter than within the mean-field approach. This is a 


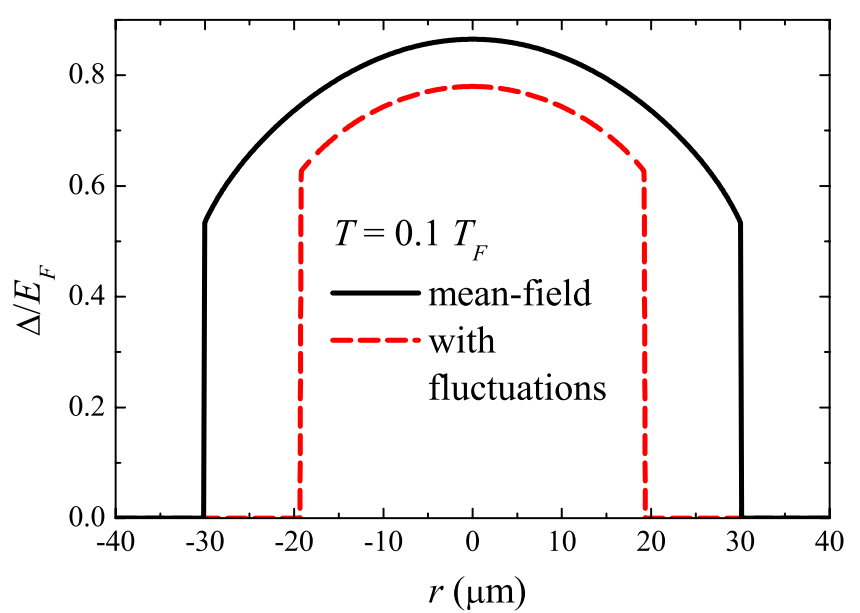

FIG. 2. (Color online) 3D distribution profiles along the lateral direction for the gap parameter calculated without (solid curve) and with (dashed curve) Gaussian fluctuations for cold fermions in the parabolic trap at $T=0.1 T_{F}$ and $\delta N / N=0.58$.

consequence of the fact that fluctuations lower the stability of the superfluid state.

As found in Refs. [19,21,32,37,38], the NSR scheme leads to difficulties near the unitarity limit, where the fluctuation contribution to the fermion density is not small and therefore can be hardly treated as a perturbation. For example, close to the critical temperature, the NSR approach predicts a bend-over behavior of the gap parameter as a function of the temperature [21]. Parish et al. [32] showed that near unitarity, the susceptibility matrix

$$
\left\|\chi_{j k}\right\|=\left(\begin{array}{ll}
\left(\frac{\partial n_{+}}{\partial \mu_{+}}\right)_{T, \Delta, \mu_{-}} & \left(\frac{\partial n_{+}}{\partial \mu_{-}}\right)_{T, \Delta, \mu_{+}} \\
\left(\frac{\partial n_{-}}{\partial \mu_{+}}\right)_{T, \Delta, \mu_{-}} & \left(\frac{\partial n_{-}}{\partial \mu_{-}}\right)_{T, \Delta, \mu_{+}}
\end{array}\right)
$$

within the NSR scheme is not positive semidefinite. They interpreted this result as a breakdown of the NSR treatment. However, this breakdown occurs when the fluctuation contributions are sufficiently high—at temperatures close to $T_{c}$ for a uniform Fermi gas at unitarity. Also the bend-over behavior of the gap parameter predicted by Taylor et al. [21] occurs near the critical temperature for a balanced uniform Fermi gas $T_{c} \approx 0.23 T_{F}[26]$.

The fluctuation contribution to the fermion density strongly falls down with decreasing temperature. The experiments of the MIT group $[10,11]$ are performed for lower temperatures $T \leqq 0.1 T_{F}$, at which the fluctuation contribution to the density is smaller than at $T_{c}$ for a balanced uniform gas. In this connection, we can expect that the fluctuation contribution to the fermion density is relatively small with respect to the mean-field contribution, and therefore the extended NSR scheme can be applicable to the experimental conditions of Refs. [8-13]. To verify this assumption, we study the behavior of the susceptibility matrix for cold fermions in a trap.

The positive semidefinite matrix means that its eigenvalues are non-negative. The eigenvalues $\lambda_{1}$ and $\lambda_{2}$ of the ma-

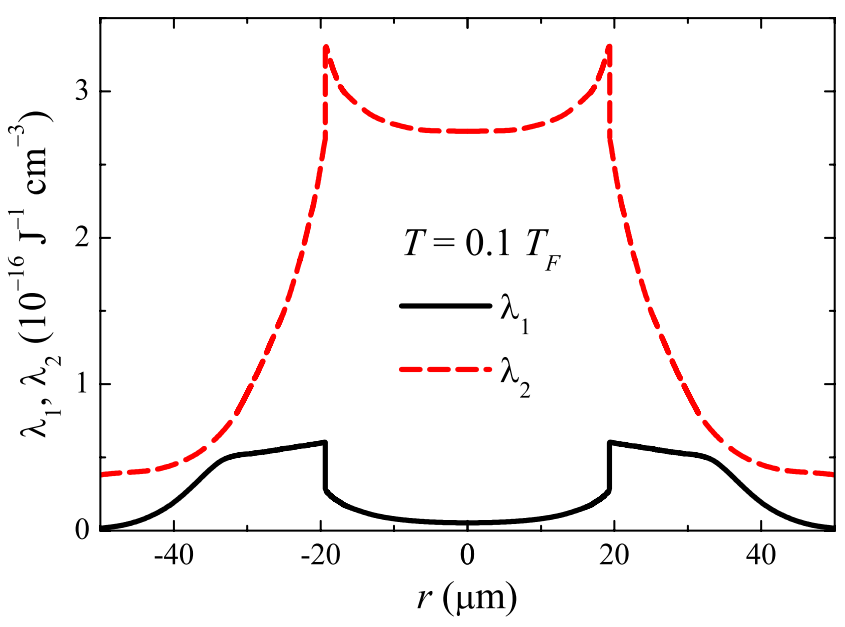

FIG. 3. (Color online) 3D distribution profiles for the eigenvalues $\lambda_{1}, \lambda_{2}$ of the susceptibility matrix for imbalanced interacting fermions in the parabolic trap at $T=0.1 T_{F}$ and $\delta N / N=0.58$.

trix (7) can be expressed through the derivatives of the total density $n$ and of the density difference $\delta n$ using as the independent variables the averaged chemical potential $\mu$ and the imbalance potential $\zeta$,

$$
\begin{aligned}
\lambda_{1,2}= & \frac{1}{4}\left\{\left(\frac{\partial n}{\partial \mu}\right)_{T, \Delta, \zeta}+\left(\frac{\partial(\delta n)}{\partial \zeta}\right)_{T, \Delta, \mu}\right. \\
& \left.\mp \sqrt{\left[\left(\frac{\partial n}{\partial \mu}\right)_{T, \Delta, \zeta}-\left(\frac{\partial(\delta n)}{\partial \zeta}\right)_{T, \Delta, \mu}\right]^{2}+4\left(\frac{\partial n}{\partial \zeta}\right)_{T, \Delta, \mu}^{2}}\right\} .
\end{aligned}
$$

As follows from the relation

$$
\left(\frac{\partial n}{\partial \zeta}\right)_{T, \Delta, \mu}=\left(\frac{\partial(\delta n)}{\partial \mu}\right)_{T, \Delta, \zeta},
$$

in the limit of a vanishing imbalance, when $\delta n=0,\left(\frac{\partial n}{\partial \zeta}\right)_{T, \Delta, \mu}$ $=0$. Therefore, in this case the eigenvalues of the susceptibility matrix are reduced to the derivatives $\left(\frac{\partial n}{\partial \mu}\right)_{T, \Delta, \zeta}$ and $\left(\frac{\partial(\delta n)}{\partial \zeta}\right)_{T, \Delta, \mu}$.

In order to check whether the susceptibility matrix is positive semidefinite at the conditions of the experiments under consideration $[10,11]$, we analyze the eigenvalues of the susceptibility matrix as a function of the radius using the values of parameters relevant to the experiment [10]. The eigenvalues are calculated taking into account Gaussian fluctuations about the saddle point. The $3 \mathrm{D}$ distribution profiles for the eigenvalues of the susceptibility matrix (8) are shown in Fig. 3.

The susceptibility matrix eigenvalues, as well as the gap parameter and the density profiles, behave discontinuously at the phase boundary. We see that in the stable superfluid and normal states, both $\lambda_{1}$ and $\lambda_{2}$ are positive. This result shows that for the experimental conditions of Refs. $[10,11]$, the NSR scheme extended to the imbalanced case provides a reasonable interpretation of the phase separation of a trapped Fermi gas. 
Currently, the temperature in the experiment is determined by fitting to the tails of the remnant thermal distributions. One of the implicit assumptions is a good thermalization between the superfluid and the thermal cloud, and this assumption has recently been under further investigation [39]. Owing to fluctuations, the density profile is rather sensitive to temperature variations at low temperatures $T \ll T_{F}$. The good agreement between the temperature-dependent density profiles calculated here and the experimental density profiles allows us to estimate the temperature of the superfluid core.

In conclusion, we have investigated the density profiles of a phase separated imbalanced Fermi gas in an anisotropic parabolic trap, taking into account Gaussian fluctuations near the saddle point at a nonzero temperature. Fluctuations lead to a smoothing of the density profiles and to a decrease of the radius of the superfluid core with respect to those predicted by the mean-field approximation. The calculated density profiles are in good agreement with the experimental density profiles observed in Ref. [10] and their temperature dependence enables temperature determination. The present approach allows for a much more convincing interpretation of the experimental results on the phase separation of imbalanced fermions with respect to the mean-field approximation.

We are grateful to W. Ketterle, M. Wouters, D. Lemmens, J. O. Indekeu, M. K. Oberthaler, E. Timmermans, and H. T. C. Stoof for valuable discussions. This work has been supported by the FWO-V Contracts No. G.0356.06, No. G.0115.06, No. G.0435.03, and No. G.0306.00, the WOG Contract No. WO.025.99N, and the NOI BOF UA 2004.
[1] R. Casalbuoni and G. Nardulli, Rev. Mod. Phys. 76, 263 (2004).

[2] Yu. A. Litvinov et al., Phys. Rev. Lett. 95, 042501 (2005).

[3] E. Braaten and H.-W. Hammer, Phys. Rep. 428, 259 (2006).

[4] G. Labeyrie, F. Michaud, and R. Kaiser, Phys. Rev. Lett. 96, 023003 (2006).

[5] P. F. Bedaque, Nucl. Phys. A 697, 569 (2002).

[6] M. G. Alford, J. A. Bowers, and K. Rajagopal, Phys. Rev. D 63, 074016 (2001).

[7] A. Sedrakian and U. Lombardo, Phys. Rev. Lett. 84, 602 (2000).

[8] M. W. Zwierlein, A. Schirotzek, C. H. Schunck, and W. Ketterle, Science 311, 492 (2006).

[9] M. W. Zwierlein, C. H. Schunck, A. Schirotzek, and W. Ketterle, Nature (London) 442, 54 (2006).

[10] Y. Shin, M. W. Zwierlein, C. H. Schunck, A. Schirotzek, and W. Ketterle, Phys. Rev. Lett. 97, 030401 (2006).

[11] G. B. Partridge, W. Li, R. I. Kamar, Y.-A. Liao, and R. G. Hulet, Science 311, 503 (2006).

[12] G. B. Partridge, W. Li, Y. A. Liao, R. G. Hulet, M. Haque, and H. T. C. Stoof, Phys. Rev. Lett. 97, 190407 (2006).

[13] G. B. Partridge, W. Li, Y. A. Liao, and R. G. Hulet, J. Low Temp. Phys. 148, 323 (2007).

[14] T. N. De Silva and E. J. Mueller, Phys. Rev. A 73, 051602(R) (2006).

[15] M. Haque and H. T. C. Stoof, Phys. Rev. A 74, 011602(R) (2006).

[16] M. Haque and H. T. C. Stoof, Phys. Rev. Lett. 98, 260406 (2007).

[17] F. Chevy, Phys. Rev. Lett. 96, 130401 (2006).

[18] N. Yoshida and S.-K. Yip, Phys. Rev. A 75, 063601 (2007).

[19] A. Perali, P. Pieri, L. Pisani, and G. C. Strinati, Phys. Rev. Lett. 92, 220404 (2004).

[20] Q. Chen, Y. He, C.-C. Chien, and K. Levin, Phys. Rev. B 75, 014521 (2007).

[21] E. Taylor, A. Griffin, N. Fukushima, and Y. Ohashi, Phys. Rev.
A 74, 063626 (2006).

[22] P. Nozières and S. Schmitt-Rink, J. Low Temp. Phys. 59, 195 (1985)

[23] H. Hu, X.-J. Liu, and P. D. Drummond, Europhys. Lett. 74, 574 (2006).

[24] H. Hu, P. D. Drummond, and X.-J. Liu, Nat. Phys. 3, 469 (2007).

[25] H. Hu, X.-J. Liu, and P. D. Drummond, Phys. Rev. A 77, 061605(R) (2008).

[26] C. A. R. Sá de Melo, M. Randeria, and J. R. Engelbrecht, Phys. Rev. Lett. 71, 3202 (1993).

[27] J. R. Engelbrecht, M. Randeria, and C. A. R. Sá de Melo, Phys. Rev. B 55, 15153 (1997).

[28] R. B. Diener, R. Sensarma, and M. Randeria, Phys. Rev. A 77, 023626 (2008).

[29] P. F. Bedaque, H. Caldas, and G. Rupak, Phys. Rev. Lett. 91, 247002 (2003)

[30] D. J. Thouless, Ann. Phys. 10, 553 (1960).

[31] J. Tempere, S. N. Klimin, J. T. Devreese, and V. V. Moshchalkov, Phys. Rev. B 77, 134502 (2008).

[32] M. M. Parish, F. M. Marchetti, A. Lamacraft, and B. D. Simons, Nat. Phys. 3, 124 (2007).

[33] T. K. Koponen, T. Paananen, J.-P. Martikainen, and P. Törmä, Phys. Rev. Lett. 99, 120403 (2007).

[34] W. Zhang and L.-M. Duan, Phys. Rev. A 76, 042710 (2007).

[35] C.-H. Pao, S.-T. Wu, and S.-K. Yip, Phys. Rev. B 73, 132506 (2006); 74, 189901(E) (2006).

[36] D. E. Sheehy and L. Radzihovsky, Phys. Rev. B 75, 136501 (2007).

[37] C.-C. Chien, Q. Chen, Y. He, and K. Levin, Phys. Rev. Lett. 98, 110404 (2007).

[38] Y. He, C.-C. Chien, Q. Chen, and K. Levin, Phys. Rev. B 76, 224516 (2007).

[39] B. V. Schaeybroeck and A. Lazarides, Phys. Rev. Lett. 98, 170402 (2007). 